\title{
国内作者撰写SCI论文的 常见误区
}

胡志德

内蒙古医科大学附属医院检验科

从全球范围来看，各个地区、各个民族都会有 自己独特的文化传承, 这种文化传承在很大程度上 会影响人们的言行和思维习惯。比如: 如果有朋友 夸自己的小孩聪明, 中国人往往回复以 “过奖了”、 “哪里啊”之类的很谦虚的词句, 而西方人更喜欢用 “Thank you”一笑而过。以 SCI 为代表的科学论文 发表、传播与评价体系是由西方首先发起并主导的, 因此这套体系中很多固有的思维模式和行为习惯仍 然是西式的。国内作者想发表 SCI 论文, 就必须摒 弃一些中式的思维, 学会用西方学者, 特别是欧美 学者的思维方式和习惯来撰写论文。

在过去的几年里, 笔者时常帮助一些朋友修改 论文, 也为二十余本 SCI 杂志评阅了一百余篇论文, 每次审阅到、或者修改到一些国内学者的论文, 总能 发现其中或多或少存在一些中式的表达方式和思维 模式。这些中式的表达方式和思维习惯, 可能与国 内作者长期阅读中文论文有关。实际上, 中文论文与 SCI 论文的写作风格、写作理念完全不同。如果把写 作中文论文的思维方式和习惯用到 SCI 论文写作上, 轻则会闹笑话, 重则导致拒稿。在此, 笔者结合自己 长期修改和审阅 SCI 论文的经历, 浅析一些国内作 者写作 SCI 论文的常见误区，或者说写作陋习。

\section{喜欢用 $A B C$ 组来指代各个分组}

将研究对象分为 A 组、 $\mathrm{B}$ 组和 $\mathrm{C}$ 组的表达方式 在中文论文中随处可见, 甚至可以说俯拾皆是。但 纵观国际上高水平的杂志, 几乎从来不会用 $\mathrm{ABC}$ 组
的表达方式。有的国内作者辩解道: $\mathrm{ABC}$ 组的表达 方式很简单明了啊, 自己写起来十分方便。殊不知, 论文的写作不能定位于 “自己方便”, 而应该是 “读 者方便”。试想, 读者想了解一篇论文的核心内容, 最快的方式是就是看图表。但如果图表中充斥的文 字都是 $\mathrm{ABC}$ 组, 又如何能快速掌握文章的核心内容? 更重要的是, 一旦使用了 $\mathrm{ABC}$ 组之类的表达后, 就 会强迫读者时刻牢记 $\mathrm{ABC}$ 组所谓何物。如果在阅 读途中突然忘了 $\mathrm{ABC}$ 组指代的内容, 就不得不回头 在材料和方法中去寻找相关说明, 这显然会影响阅 读体验。正确的表达方式应该是直接用分组要素进 行命名, 比如肝炎组、IL-2 敲除组等。这种表达方 式直截了当, 无需读者再进行信息转换。

\section{不喜欢用图展示数据}

中文杂志的特点是喜欢用数字展示结果。比如 描述肝癌患者和健康个体的血清 IL-2 水平, 中文 论文的做法就是在文章结果部分写一句话: 肝癌患 者的血清 IL-2 水平为 $35 \pm 15 \mathrm{pg} / \mathrm{ml}$, 健康对照组为 $12 \pm 6 \mathrm{pg} / \mathrm{ml}$, 二者的差异具有统计学意义 $(\mathrm{t}=3.84$, $\mathrm{p}=0.01)$ 。这种结果呈现方式最大的问题在于重点不 够凸显, 或者说重要信息被文字淹没了。试想, 如果 肝癌患者血清 IL-2 水平增高是一个极为关键的发现, 作者就应该进行凸显, 以便读者在第一时间掌握这一 关键信息。众所周知, 科学论文有三种结果呈现方式: 文字、表格和图片。其中, 视觉冲击力最强的就是图 片。因此, 此处以柱状图或点状图的方式来呈现肝 
癌组和健康对照组的血清 IL-2 水平才是较为明智的 选择, 即便这一结果本身可以 “凑合” 着放在表格中。 实际上，在高水平的论文中，图表往往是独立成文的。 读者不需要借助任何文字, 就可以像看 “连环画” 样把握住研究的全部核心内容。

也许有人会说，图片不够精确，从图片上面理 解的数据会有一定误差, 文字表达具体的数字才够 精确。笔者并不同意这一观点, 因为: 医学研究都是 通过样本去推断总体, 理论上讲, 即便实验条件相同, 每次得到的结果也不尽相同，有微小的误差本身是 允许的。以图片呈现结果虽然不够精确, 但是很多 结果, 本身就不需要太精确。在上述案例中, 肝癌 组的 IL-2 均值到底是 $35 \mathrm{pg} / \mathrm{ml}$ 还是 $34 \mathrm{pg} / \mathrm{ml}$ 本身 就是一个不重要的信息。退一万步讲, 即便这个信息 必须精确表示，也完全可以在柱状图或点状图中标 识出均值和标准差。

\section{废话太多，没有把握住重点}

如果一篇论文中包含了大量读者不感兴趣的信 息, 读者就会读得很累, 最终把握不住重点。因此, 医学论文写作的核心原则之一就是简洁明了。很多国 内学者, 总是抱着一副凑字数的心态去写作论文, 论 文中包含了大量冗余信息就不足为奇。这种现象在讨 论中极为常见。很多论文的讨论部分完全就是知识背 景的介绍, 没有关于研究结果的解读。实际上, 讨论 部分最重要的凝练出研究的创新性、局限性、现实意 义和科学价值, 可以适当介绍相关研究, 但最终必 须落实到自己的研究结果的解读之上。一般而言, 可 以通过以下表达方式将相关研究和自己的研究 “串联” 起来: “我们在前述研究的基础上发现了***, 进一步 证明了***”; “与此前的研究相比, 我们的研究具有 以下优势”。如此等等, 核心是要将讨论的重点落 实到自己的研究，而非简单的背景介绍。

在写材料和方法的过程中，简明扼要极为重要。 虽然研究的方法步骤很多, 但读者往往只关心其中 的部分信息。对于作者而言, 只需要集中笔墨把这 些细节介绍清楚即可, 至于其它细节, 完全可以一笔 带过。笔者曾修改过一篇文章，作者探讨某标记物 在肺癌预后评估中的价值, 材料方法中写道: 所有 肺癌患者入组后, 都接受某化疗药物的治疗。具体
过程为: 将某化疗药物溶于 $500 \mathrm{ml}$ 生理药水中, 然 后静脉滴注, 两小时内完成。这就是典型的无用信 息! 静脉滴注到底是 $500 \mathrm{ml}$ 生理盐水滴注两小时还 是 $300 \mathrm{ml}$ 其它液体滴注一小时, 显然不会影响研究 结果, 也不是读者关心的内容。因此, 作者只需要说 明所有患者入组后静脉滴注某化疗药物治疗, 并说 明治疗剂量和时长即可。

\section{标题没有信息量}

很多国内的作者在写作 SCI 论文的标题时, 仍 然遵循国内杂志的风格, 给论文起个大而宽的标题。 比如: 脑卒中患者 D-二聚体检测的临床价值; 维生 素 D 对 A549 细胞调亡的影响。翻译成英文, 就是： Clinical value of D-dimer in stroke patients; Effect of vitamin D on the apoptosis of A549 cell line。这类标 题最大的问题在于: 不够具体, 无法吸引住读者。以 Clinical value of D-dimer in stroke patients 这个标题 为例, 最大的问题是读者无法从标题中得知研究的主 要结果和结论, 或者说无法得知来研究的来龙去脉。 如果将标题改为 Increased plasma D-dimer on admission predicts lower over survival in adults ischemic stroke patients: a prospective and 5-year follow up cohort study with 5900 subjects, 给人的感觉就不一样了。 研究的主要发现 (D-二聚体增高的成年缺血性脑卒中 患者总体生存率较低)、设计类型 (前瞻性队列研究) 和优势 (样本量大、随访时间长) 就跃然纸上了。实 际上，论文发表并不是科研的最后一步，发表后的曝 光也极端重要。试想, PubMed 里面的文献多如牛毛, 读者是否会点击某一篇文章, 在很大程度上取决于文 章的标题是否清晰和有信息量。Clinical value of Ddimer in stroke patients 这种标题虽然清晰, 但是没有 信息量，也难以勾起读者的阅读兴趣。

\section{喜欢写统计值}

国内有很多杂志要求作者在交代统计学结果的时 候写明统计值 ( $\mathrm{Z}$ 值、 $\mathrm{t}$ 值、卡方值等)。受这一规定 的影响, 很多作者想当然地认为写 SCI 论文也应该写 明具体的统计值。实际上，目前 SCI 杂志基本不会要 求作者写统计值。之所以说基本二字，一是因为写统 


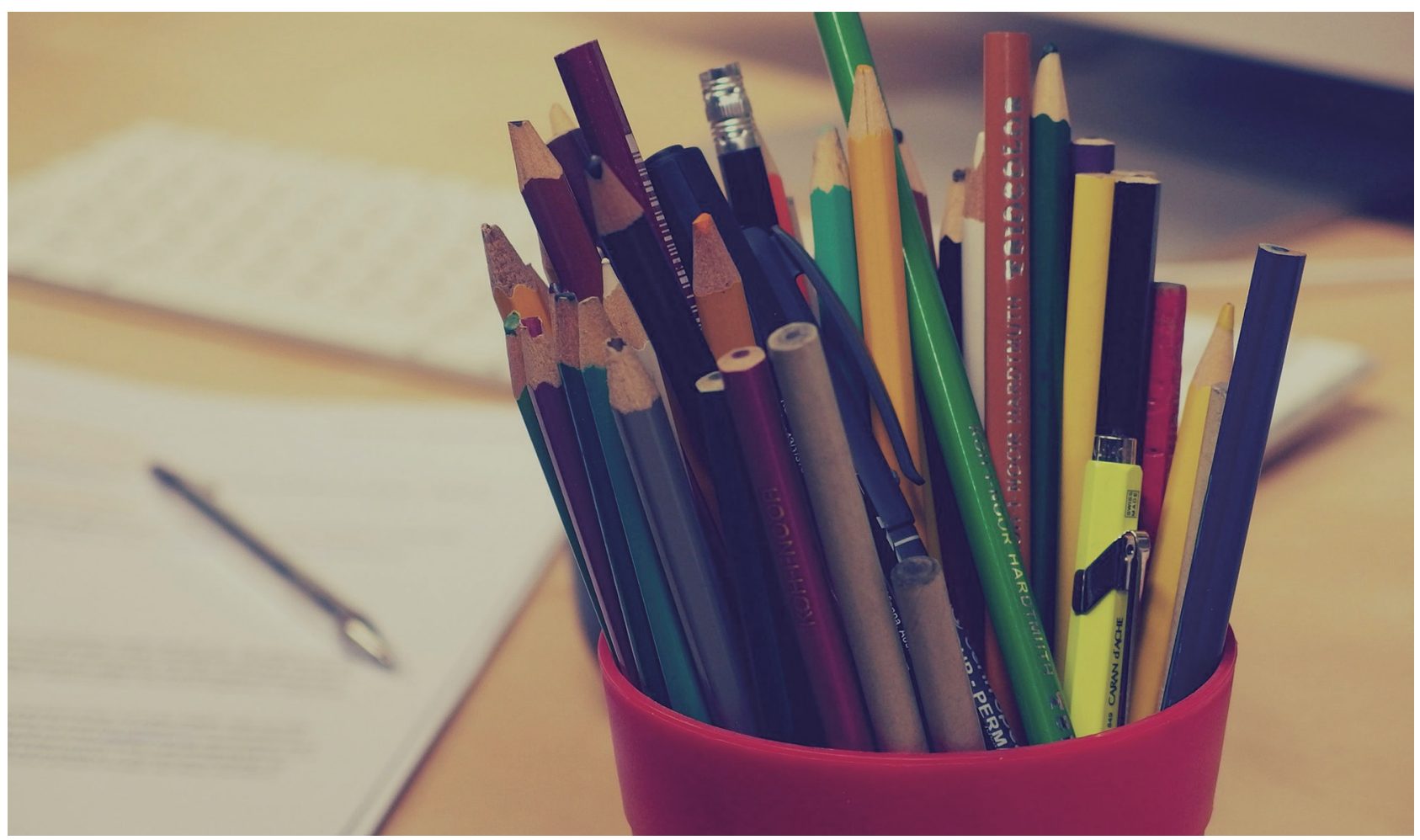

计值的 SCI 论文少之又少, 且多出自国人之手; 二是 因为主流的、顶级的杂志从不要求作者报告统计值。

从统计学的角度而言, 人们最关心的是效应量 的大小和结果属于抽样误差的概率, 前者是以差值 ( 比如均数的差值、中位数的差值等) 或比值 (比如 优势比、风险比等) 的形式较为直观地呈现出来, 后者则是 $\mathrm{P}$ 值, 统计值其实没有传递任何作者关心 的信息。有的读者可能会说, 标明统计值其实是想 说明自己用的统计方法是正确的, 比如写了 $\mathrm{t}$ 值意味 着这是用了 $\mathrm{t}$ 检验, 卡方值则意味着作者用了卡方检 验。这种想法虽然有一定道理, 到不符合目前主流 的做法。目前, 读者或审稿人判断作者的统计学方 法是否正确, 主要还是基于 statistical analysis 中的 描述。而撰写 statistical analysis 这部分内容时, 往 往需要作者把握住 “火候” : 写得太简单了, 读者看 不出作者的统计学方法是否正确; 写得太复杂了, 会 显得很啰嗦, 影响阅读体验。试想, 如果一个作 者写的是: 本研究中年龄、谷草转氨酶是服从正态 分布的, 且方差齐, 所以用独立样本 $\mathrm{t}$ 检验进行两 组均值的比较; 血糖和白蛋白方差不齐, 所以采用 Mann-Whitney 比较这两个指标在两组中的分布特点 是否具有显著差异。这种写法虽然专业上没有错误, 但是比较啰嗦, 还不如直接写一句: Continuous variables between two groups were compared with student's t test or Mann-Whitney U test, if approximate. 这其实是一种广泛使用的表达方式。作者能写 出这样的词句, 说明其已经注意了是否正态分布的问 题, 一般也就默认了作者所用的统计学方法是正确 的。所以, 论文写作中没有必要以报告统计值的方 式去证明自己所有的统计学方法是正确的。

\section{不会在前言中用转折语气}

在前言中用转折语气是一种国际上十分流行的 做法, 其目的是引出本研究的创新性。毫无疑问, 没有创新性的论文是很难发表的。评阅论文的专家 不见得一定是本领域的大同行, 因此可能无法准确 地凝练出研究的创新性。在此情况下, 比较明智的 做法就是作者自己凝练创新性, 并以转折语气的方 式在前言中呈现。

笔者曾遇见过一篇论文, 核心内容是评价某循 环 microRNA 诊断肺癌。在前言中, 作者先花一些 笔墨介绍了三方面的内容: 肺癌的危害性、早期诊 断肺癌的必要性以及现行手段的不足、循环 microRNA 的特点和定义。然后话锋一转, 直接在后面写 
了句 “本研究旨在探讨血清 microRNA 诊断肺癌的 价值”。这种谋篇布局方式在思维上存在逻辑跳跃, 读者会产生不解: 已经发现的 microRNA 有上千种, 作者为何认定 microRNA 可以辅助肺癌的诊断? 实 际上, 作者之所以认定 microRNA 可以辅助诊断肺 癌, 主要是受了既往研究的启示, 确实已经有好几 项研究探讨了循环 microRNA 诊断肺癌的价值。但 作者在前言中刻意不提及这几项研究, 以凸显自己 研究的创新性。殊不知, 这种行为属于典型的学术 不诚实, 不尊重他人的研究成果。更重要的, 试想: 如果审稿专家刚好做过 microRNA 诊断肺癌的研究, 但却发现作者并未引用自己的论文，会作何感想?

正确的方式应该是回顾既往的相关研究取得的 进展, 然后凝练出既往的不足, 最后以一个 however 或 although 引导的转折语气来凸显自己研究的 创新性。比如: Some studies have investigated the diagnostic accuracy of plasma microRNA for lung cancer. However, all of the published studies, to our knowledge, are retrospective design and their sample sizes are $<200$. Here, we performed a prospective studies with 500 subjects to evaluate the diagnostic accuracy of plasma microRNA for lung cancer。这 种表达方式既没有回避相关研究, 同时也凸显了本 研究的创新性, 即前瞻性研究、样本量大。实际上, 医学研究本身允许百家争鸣, 即便是同一个 topic, 也需要不断开展不同的研究, 从各个方面进行论证。 所以, 论文要发表的前提是自己的研究在某个局部 有创新性, 而并不强制要求在全局上具有创新性。

\section{不会写局限性}

撰写中文论文基本不用写局限性, 因为一旦写了 局限性容易被审稿专家揪住, 作为拒稿的理由之一。
但绝大部分 SCI 论文, 都会在讨论部分的倒数第二 段介绍文章的局限性。有读者可能会问, 为什么 SCI 论文要撰写局限性呢? 因为: 第一, 任何研究都是有 局限性的, 于作者而言, 有义务让读者全面和深入 地了解文章的优势和缺陷, 不能只报喜不报忧; 第 二, 也是最为重要的一点, 任何研究都是有局限性 的, 即便作者不写, 审稿人也能看出来。但是审稿 人在审稿意见中写局限性和读者自己的论文中剖析 局限性, 给编辑和主编造成的冲击是完全不一样的。 审稿人指出了局限性, 主编或编辑就会认为这个局限 性是个应该严肃对待的问题; 但读者自己归纳出了局 限性, 主编则更倾向于认为作者信心十足, 研究虽 有瑕疵, 但瑕不掩瑜。通常, 审稿专家往往不会在 审稿意见中重复指出作者已经指出的局限性, 除非该 局限性不可容忍。因此, 恰当好处地指出研究的局 限性其实是一次避重就轻、展示自信的机会。

\section{结束语}

SCI 杂志的稿件评审是一件很 “微妙” 的事情, 拒稿和接受往往就在一念之间。从理论上讲, 稿件 是否接受取决于四大要素: 研究是否具有临床价值、 是否具有创新性、设计是否合理以及论文撰写是否 流畅清晰, 但不得不说的是: 一些四大要素之外的 因素会在很大程度上影响审稿人的心情, 并最终影 响判断。比如: 如果作者用的标题是 Clinical value of D-dimer in stroke patients, 审稿人往往会认为作 者不够专业。既然一篇文章出自一个不够专业的团队, 审稿人就会感觉文章可能不可靠, 必须对其进行严 格的审阅, 在模棱两可之时审稿人可能更倾向于拒 稿。所以, 做好每一个细节, 尽量遵循西方学者的 思维模式和表达方式, 给审稿人留下良好的印象, 才是提高 SCI 命中率的关键。 